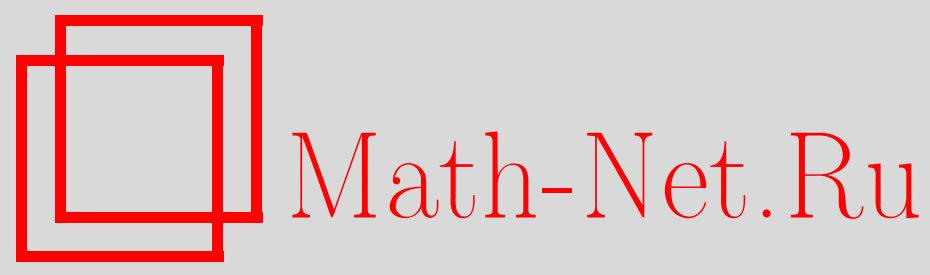

Ю. М. Арлинский, Замкнутые секториальные формы и однопараметрические полугруппы сжатий, Матем. заметки, 1997, том 61, выпуск 5, 643-654

DOI: https://doi.org/10.4213/mzm1545

Использование Общероссийского математического портала Math-Net.Ru подразумевает, что вы прочитали и согласны с пользовательским соглашением http://www.mathnet.ru/rus/agreement

Параметры загрузки:

IP : 52.87 .193 .239

26 апреля 2023 г., 14:48:39 


\title{
ЗАМКНУТЫЕ СЕКТОРИАЛЬНЫЕ ФОРМЫ И ОДНОПАРАМЕТРИЧЕСКИЕ ПОЛУГРУППЫ СЖАТИЙ
}

\author{
Ю.М. Арлинский
}

Пусть $s[u, v]$ - замкнутая полуторалинейная секториальная форма с вершиной в нуле, полууглом $\alpha \in[0, \pi / 2)$ и плотной областью определения $\mathscr{D}(s)$ в гильбертовом пространстве $H, S-m$-секториальный оператор, ассоциированный с формой $s, S_{R}$-его "реальная часть", $T(t)=\exp (-t S)$ - голоморфная в секторе $|\arg t|<\pi / 2-\alpha$ полугруппа сжатий с производящим оператором $-S$.

В работе охарактеризована форма $s$ в терминах полугруппы $T(t)$. Так, в частности, доказана эквивалентность следующих условий: 1) $u \in \mathscr{D}(s) ; 2)$ функция $\|T(t) u\|$ дифференцируема в нуле; 3$)$ функция $(T(t) u, u)$ дифференцируема в нуле.

Библиографоия: 10 названий.

1. Введение. Пусть $H$ - комплексноегильбертово пространство со скалярным произведением $(\cdot, \cdot)$ и нормой $\|\cdot\|, I$ - единичный оператор в $H, s[u, v]$ - полуторалинейная (линейная по первому аргументу и антилинейная по второму) форма с плотной в $H$ областью определения $\mathscr{D}(s), s[u]=s[u, u]$ - соответствующая квадратичная форма.

Предположим, что $s$ - замкнутая секториальная форма с вершиной в нуле и полууглом $\alpha \in[0, \pi / 2)[1],[2]$, т.е.

а) числовая область формы $W(s)=\{s[u]: u \in \mathscr{D}(s),\|u\|=1\}$ есть подмножество сектора $\Theta(\alpha)=\{z \in C:|\arg z| \leqslant \alpha\}$

б) если $\left\{u_{n}\right\} \subset \mathscr{D}(s)$,

$$
\lim _{n \rightarrow \infty} u_{n}=u, \quad \lim _{n \rightarrow \infty} s\left[u_{n}-u_{m}\right]=0
$$

то $u \in \mathscr{D}(s)$ и $\lim _{n \rightarrow \infty} s\left[u-u_{n}\right]=0$.

Как известно [1, первая теорема о представлении], с формой $s$ ассоциирован $m$-секториальньй оператор $S$ с вершиной в нуле и полууглом $\alpha$, т.е.

а) $(S x, v)=s[x, v] \forall x \in \mathscr{D}(S), \forall v \in \mathscr{D}(s)$;

б) область определения $\mathscr{D}(S)$ оператора $S$ плотна в $H$ и является ядром формы $s$;

в) резольвентное множество $\rho(S)$ оператора $S$ непусто (содержит $\operatorname{Ext} \Theta(\alpha))$;

г) для любого $x \in \mathscr{D}(S)$ выполняется неравенство $|\operatorname{Im}(S x, x)| \leqslant \operatorname{tg} \alpha \operatorname{Re}(S x, x)$.

Работа выполнена при частичной поддержке Международной соросовской программы в области точных наук (ISSEP), грант APU051009. 
Тот факт, что $\mathscr{D}(S)$ является ядром формы $s$ можно выразить соотношением

$$
\inf \left\{\operatorname{Re} s[u-x]+\|u-x\|^{2}, x \in \mathscr{D}(S)\right\}=0 \quad \forall u \in \mathscr{D}(s)
$$

Пусть $s^{*}[u, v]=\overline{s[v, u]}, u, v \in \mathscr{D}(s)$ сопряженная форма. Тогда с $s^{*}$ ассоциирован сопряженньй оператор $S^{*}[1]$.

Обозначим $s_{R}[u, v]=\left(s[u, v]+s^{*}[u, v]\right) / 2$. Форма $s_{R}$ является замкнутой и неотрицательной, с ней ассоциирован неотрицательный самосопряженный оператор $S_{R}, \mathscr{D}\left(S_{R}\right)-$ ядро формы $s$ и по второй теореме о представлении [1] выполняется $\mathscr{D}(s)=\mathscr{D}\left(S_{R}^{1 / 2}\right)$.

Оператор $-S$ является (см. [1], [2]) инфинитезимальным производящим оператором сильно непрерывной однопараметрической полугруппы сжатий

$$
T(t)=\exp (-t S), \quad t \geqslant 0
$$

имеющей голоморфное сжимающее продолжение в область $\operatorname{Int} \Theta(\pi / 2-\alpha)$.

При этом

$$
\begin{gathered}
\mathscr{D}(S)=\left\{x \in H: \lim _{t \rightarrow 0} t^{-1}(I-T(t)) x \text { существует }\right\} \\
S x=\lim _{t \rightarrow 0} t^{-1}(I-T(t)) x .
\end{gathered}
$$

Область определения $\mathscr{D}(S)$ может быть также охарактеризовано следующим обра$30 \mathrm{OM}[3]$ :

$$
\mathscr{D}(S)=\left\{x \in H: \sup _{t>0}\left\|t^{-1}(I-T(t)) x\right\|<\infty\right\}
$$

В настоящей работе в терминах полугрупшы $T(t)$ охарактеризована форма $s$.

Примем обозначения: $\mathscr{L}\left(H_{1}, H_{2}\right)$ - банахово пространство всех линейных ограниченных операторов, определенных на гильбертовом пространстве $H_{1}$ со значениями в гильбертовом пространстве $H_{2}, \mathscr{L}(H)=\mathscr{L}(H, H) ; \operatorname{Ker} T, \mathscr{R}(T)$ - соответственно ядро и область значений оператора $T, T_{R}=\left(T+T^{*}\right) / 2$ для $T \in \mathscr{L}(H), D_{T}=\left(I-T^{*} T\right)^{1 / 2}$, $\mathscr{D}_{T}=\overline{\mathscr{R}\left(D_{T}\right)}$ для сжатия $T \in \mathscr{L}\left(H_{1}, H_{2}\right), \sigma(T)$ и $\rho(T)$ - соответственно спектр и резольвентное множество оператора $T$.

Мы докажем следующие теоремы.

Теорема 1. Следующие условия әквивалентны:

1) $u \in \mathscr{D}(s)$;

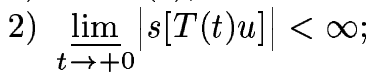

3) $\sup \left\{t^{-1}|((I-T(t)) u, u)|, t>0\right\}<\infty$;

4) $\sup \left\{\operatorname{Re}\left(t^{-1}(I-T(t)) u, u\right), t>0\right\}<\infty$;

5) существует производная $\left.\frac{d}{d t}(T(t) u, u)\right|_{t=0}$;

6) существует производная $\left.\frac{d}{d t}(\|T(t) u\|)\right|_{t=0}$. 
Если и, v $\mathscr{D}(s)$, то справедливы равенства

$$
\begin{gathered}
\left.\frac{d}{d t}(\|T(t) u\|)\right|_{t=0}=-\frac{s_{R}[u]}{\|u\|} ; \\
\left.\frac{d}{d t}(T(t) u, v)\right|_{t=0}=-s[u, v] ; \\
\left.\frac{d}{d t}(T(t) u, T(t) v)\right|_{t=0}=-2 s_{R}[u, v] ; \\
\|(I-T(t)) u\|=o(\sqrt{t}), \quad t \rightarrow+0 .
\end{gathered}
$$

Tеорема 2. Для любого $t \in \operatorname{Int} \Theta(\pi / 2-\alpha)$ справедливы равенства

$$
\mathscr{R}\left(D_{T(t)}\right)=\mathscr{R}\left(D_{T^{*}(t)}\right)=\mathscr{R}\left(\left(I-T_{R}(t)\right)^{1 / 2}\right)=\mathscr{R}\left(S_{R}^{1 / 2}\right) .
$$

2. Предварительные результаты. Имеют место соотношения [1], [2]

$$
\begin{gathered}
s[u, v]=\left((I+i G) S_{R}^{1 / 2} u, S_{R}^{1 / 2} v\right), \quad u, v \in \mathscr{D}(s), \\
S=S_{R}^{1 / 2}(I+i G) S_{R}^{1 / 2},
\end{gathered}
$$

где $G=G^{*} \in \overline{\mathscr{L}} \overline{\left(\mathscr{R}\left(S_{R}^{1 / 2}\right)\right)},\|G\| \leqslant \operatorname{tg} \alpha$.

Из (6) следует $\operatorname{Ker} S=\operatorname{Ker} S^{*}=\operatorname{Ker} S_{R}$, поэтому для простоты в дальнейшем предположим, что $\operatorname{Ker} S_{R}=\{0\}$.

Приведем ряд результатов, используемых в последующем.

Теорема 3 [1, гл. 6, теорема 1.16]. Пусть $s$ - замкнутая секториальная форма, $\left\{u_{n}\right\} \subset \mathscr{D}(s), \lim u_{n}=u$ и последовательность $\left\{s\left[u_{n}\right]\right\}$ ограничена. Тогда $u \in \mathscr{D}(s)$.

ПРЕДЛОЖЕНИЕ 1. Справедливы равенства

$$
\mathscr{R}\left(S_{R}^{1 / 2}\right)=\left\{x \in H: \sup _{y \in \mathscr{D}(S)} \frac{|(x, y)|^{2}}{\operatorname{Re} s[y]}<\infty\right\}=\left\{x \in H: \sup _{y \in \mathscr{D}\left(S^{*}\right)} \frac{|(x, y)|^{2}}{\operatorname{Re} s[y]}<\infty\right\} .
$$

ДокАЗАТЕльСТво. Докажем только одно равенство. Пусть $u \in \mathscr{D}\left(S_{R}^{1 / 2}\right)$.

Из $(5)$ с учетом того, что $\mathscr{D}(S)$ ядро оператора $S_{R}^{1 / 2}$ и $\mathscr{R}\left(S_{R}^{1 / 2}\right)$ плотно в $H$, имеем

$$
\sup _{y \in \mathscr{D}(S)} \frac{\left|\left(S_{R}^{1 / 2} u, y\right)\right|^{2}}{\operatorname{Re} s[y]}=\sup _{y \in \mathscr{D}(S)} \frac{\left|\left(u, S_{R}^{1 / 2} y\right)\right|^{2}}{\left\|S_{R}^{1 / 2} y\right\|^{2}}=\|u\|^{2} .
$$

Наоборот, если справедливо $|(x, y)|^{2} \leqslant C(x) \operatorname{Re} s[y]$ для всех $y \in \mathscr{D}(S)$ с некоторой постоянной $C(x)$, то опять, принимая во внимание (5) и то, что $\mathscr{D}(S)$ - ядро оператора $S_{R}^{1 / 2}$, получим уже для всех $y \in \mathscr{D}\left(S_{R}^{1 / 2}\right)=\mathscr{D}(s)$ выполнено $|(x, y)|^{2} \leqslant C(x)\left\|S_{R}^{1 / 2} y\right\|^{2}$. Отсюда $\left|\left(x, S_{R}^{-1 / 2} z\right)\right|^{2} \leqslant C(x)\|z\|^{2} \forall z \in \mathscr{R}\left(S_{R}^{1 / 2}\right)$. По теореме Рисса $x \in \mathscr{D}\left(S_{R}^{-1 / 2}\right)=\mathscr{R}\left(S_{R}^{1 / 2}\right)$. 
Из предложения 1 следует

$$
\mathscr{D}(s)=\left\{u \in H: \sup _{y \in \mathscr{D}(S)} \frac{|(S y, u)|^{2}}{\operatorname{Re} s[y]}<\infty\right\}=\left\{u \in H: \sup _{y \in \mathscr{D}\left(S^{*}\right)} \frac{\left|\left(S^{*} y, u\right)\right|^{2}}{\operatorname{Re} s[y]}<\infty\right\} .
$$

Действительно, рассмотрим определенную на $\mathscr{R}\left(S_{R}^{1 / 2}\right)$ замкнутую секториальную форму $\omega[f, g]=\left((I+i G)^{-1} S_{R}^{-1 / 2} f, S_{R}^{-1 / 2} g\right)$.

Очевидно, что с формой $\omega$ ассоциирован $m$-секториальньй оператор

$$
S^{-1}=S_{R}^{-1 / 2}(I+i G)^{-1} S_{R}^{-1 / 2} \text {. }
$$

Имеем

$$
\operatorname{Re} \omega[f]=\left\|(I+i G)^{-1} S_{R}^{-1 / 2} f\right\|^{2} \quad \forall f \in \mathscr{R}\left(S_{R}^{1 / 2}\right) .
$$

Обозначим через $W_{R}$ неотрицательньй самосопряженньй оператор, ассоциированный с формой $\omega_{R}$. Тогда $\mathscr{D}\left(W_{R}^{1 / 2}\right)=\mathscr{R}\left(S_{R}^{1 / 2}\right)=\mathscr{D}\left(\omega_{R}\right)$ и

$$
\left\|W_{R}^{1 / 2} f\right\|^{2}=\left\|(I+i G)^{-1} S_{R}^{-1 / 2} f\right\|^{2} \quad \forall f \in \mathscr{R}\left(S_{R}^{1 / 2}\right) .
$$

Поэтому $W_{R}^{1 / 2} f=U(I+i G)^{-1} S_{R}^{-1 / 2} f, f \in \mathscr{R}\left(S_{R}^{1 / 2}\right)$, где $U$ - унитарный оператор в $H$. Отсюда

$$
\begin{gathered}
(I-i G)^{-1} U^{-1} \mathscr{R}\left(S_{R}^{1 / 2}\right)=\mathscr{R}\left(S_{R}^{1 / 2}\right), \\
W_{R}^{1 / 2} f=S_{R}^{-1 / 2}(I-i G)^{-1} U^{-1} f, \quad f \in \mathscr{R}\left(S_{R}^{1 / 2}\right) .
\end{gathered}
$$

Следовательно, $\mathscr{R}\left(W_{R}^{1 / 2}\right)=\mathscr{D}\left(S_{R}^{1 / 2}\right)$. Теперь из предложения 1 следуют равенства (7).

Отметим, что предложение 1 и (7) известны для случая неотрицательных замкнутых форм (см., например, [4]-[6]).

Отнесем $T \in \mathscr{L}(H)$ к классу $C(\alpha)(\alpha \in[0, \pi / 2))$, если

$$
\|T \sin \alpha \pm i \cos \alpha I\| \leqslant 1 \text {. }
$$

Условия (8) при всех $f \in H$ эквивалентны неравенству

$$
\operatorname{tg} \alpha\left(\|f\|^{2}-\|T f\|^{2}\right) \geqslant 2|\operatorname{Im}(T f, f)| \text {. }
$$

Поэтому операторы класса $C(\alpha)$ являются сжатиями, $C(0)$ - класс всех самосопряженных сжатий.

Обозначим $\widetilde{C}=\bigcup\{C(\alpha), \alpha \in[0, \pi / 2)\}$.

Нетрудно видеть, что дробно-линейное преобразование $T=(I-S)(I+S)^{-1}$ устанавливает биективное соответствие между $m$-секториальными операторами $S$ с вершиной в нуле и полууглом $\alpha$ и операторами $T$ класса $C(\alpha)$, для которых $\operatorname{Ker}(I+T)=\{0\}$. 
TeOpema 4 [7]. Ecлu $T \in \widetilde{C}, m o$

1) подпространства $\mathscr{D}_{T}$ и $\mathscr{D}_{T^{*}}$ совпадают, $T \mid \mathscr{D}_{T}$ - принадлежит классу $C_{00}[8], T \mid H \ominus \mathscr{D}_{T}-$ самосопряжсенный унитарный оператор;

2) справедливы равенства

$$
\mathscr{R}\left(D_{T}\right)=\mathscr{R}\left(D_{T^{*}}\right)=\mathscr{R}\left(D_{T_{R}}\right)
$$

3) для того чтобы сильно непрерывная полугруппа сжатий $T(t)$ допускала голоморфное сжимающее продолжение в сектор $\operatorname{Int} \Theta(\pi / 2-\alpha)$ необходимо, чтобы для всех $t \geqslant 0$ и достаточно, чтобы при $t \in[0, \delta], \delta>0$, операторь $T(t)$ принадлежали классу $C(\alpha)$.

Из (10) в подпространстве $\mathscr{D}_{T}$ следуют равенства

$$
D_{T}=D_{T^{*}} L=D_{T_{R}} M
$$

где $L^{ \pm 1}, M^{ \pm 1} \in \mathscr{L}\left(D_{T}\right)$.

Для любого сжатия $T \in \mathscr{L}(H)$ очевидны равенства

$$
\begin{aligned}
\|(I \pm T) f\|^{2}+\left\|D_{T} f\right\|^{2} & =2\left\|\left(I \pm T_{R}\right)^{1 / 2} f\right\|^{2}, \\
\left\|\left(I \pm T^{*}\right) f\right\|^{2}+\left\|D_{T^{*}} f\right\|^{2} & =2\left\|\left(I \pm T_{R}\right)^{1 / 2} f\right\|^{2} .
\end{aligned}
$$

Из (9), (12) для $T \in C(\alpha)$ следует, что $I \pm T-m$-секториальные операторы с вершиной в нуле и полууглом $\alpha$. Из (12) получаем, что $\pm 1 \in \rho(T)$ тогда и только тогда, когда $\pm 1 \in \rho\left(T_{R}\right)$.

Принимая во внимание результаты [9] об операторных областях, из (12) и (13) получаем $\mathscr{R}(I \pm T), \mathscr{R}\left(I \pm T^{*}\right) \subseteq \mathscr{R}\left(\left(I \pm T_{R}\right)^{1 / 2}\right)$.

Из (11) при $-1 \in \rho(T)$ для $T \in \widetilde{C}$ имеем

$$
\mathscr{R}\left(D_{T}\right)=\mathscr{R}\left(D_{T^{*}}\right)=\mathscr{R}\left(\left(I-T_{R}\right)^{1 / 2}\right) .
$$

Нам понадобится один результат Ю. Л. Шмульяна.

Teорема 5 [10]. Пусть $T(\lambda) \in \mathscr{L}\left(H_{1}, H_{2}\right)$ - сжимающая оператор-функиия, аналитическая на связном $n$-мерном многообразии $\mathfrak{S}$. Тогда

1) линеаль $\mathscr{R}\left(D_{T(\lambda)}\right)$ и $\mathscr{R}\left(D_{T^{*}(\lambda)}\right)$ не зависят от $\lambda \in \mathfrak{S}$;

2) если $\lambda_{0}, \lambda_{1}, \lambda_{2}-$ точки из $\mathfrak{S}$ не обязательно различные, то оператор-функиия $T(\lambda)$ допускает представление

$$
T(\lambda)=T\left(\lambda_{0}\right)+D_{T^{*}\left(\lambda_{1}\right)} \Psi(\lambda) D_{T\left(\lambda_{2}\right)},
$$

əде $\Psi(\lambda) \in \mathscr{R}\left(\mathscr{D}_{T\left(\lambda_{2}\right)}, \mathscr{D}_{T^{*}\left(\lambda_{1}\right)}\right)$ - аналитическая в $\mathfrak{S}$ оператор-функиия.

Из теорем 4 и 5 следует 
ПРЕДЛОЖЕНИЕ 2. Пусть $S$ - m-секториальный оператор с верииной в нуле и полууглом $\alpha, T(\lambda)=\exp (-\lambda S), \lambda \in \operatorname{Int} \Theta(\pi / 2-\alpha)$. Тогда

1) линеаль $\mathscr{R}\left(D_{T(\lambda)}\right), \mathscr{R}\left(D_{T^{*}(\lambda)}\right), \mathscr{R}\left(\left(I-T_{R}(\lambda)\right)^{1 / 2}\right)$ не зависят от точки $\lambda \in \operatorname{Int} \Theta(\pi / 2-\alpha)$ и совпадают межсду собой

2) при любих $\lambda_{1}, \lambda_{2} \in \operatorname{Int} \Theta(\pi / 2-\alpha)$ и любом $f \in H$ вектор

$$
g=\int_{\lambda_{1}}^{\lambda_{2}}(I-T(\lambda)) f d \lambda
$$

лежит в линеале $\mathfrak{N}=\mathscr{R}\left(D_{T(\lambda)}\right)$ (не зависящем от $\left.\lambda\right)$.

ДокАЗАТЕЛЬСтво. 1) Если $|\arg \lambda|<\pi / 2-\alpha$, то оператор $\lambda S$ является $m$-секториальным с вершиной в нуле и полууглом $\alpha+|\arg \lambda|$. По теореме 4 оператор $T(\lambda)=\exp (-\lambda S)$ лежит в классе $C(\alpha+|\arg \lambda|)$ и $\mathscr{R}\left(D_{T(\lambda)}\right)=\mathscr{R}\left(D_{T^{*}(\lambda)}\right)$. По теореме 5 в силу голоморфности $T(\lambda)$ в Int $\Theta(\pi / 2-\alpha)$ линеалы $\mathscr{R}\left(D_{T(\lambda)}\right), \mathscr{R}\left(D_{T^{*}(\lambda)}\right)$ не зависят от $\lambda$ и, таким образом, совпадают между собой.

Обозначим $\mathfrak{N}=\mathscr{R}\left(D_{T(\lambda)}\right), \lambda \in \operatorname{Int} \Theta(\pi / 2-\alpha)$.

Как известно [1], для голоморфной полугрупшы $T(t)$ при $t>0$ справедлива оценка $\left\|T^{\prime}(t)\right\| \leqslant M t^{-1}$.

Поэтому оператор-функция $T(t)$ равномерно непрерывна на $[\delta, \infty)$ при любом $\delta>0$. Но тогда справедлива теорема об отображении спектра [3, предложение 8.5]:

$$
\exp (-t \sigma(S))=\sigma(T(t)) /\{0\}, \quad t>0
$$

По той же причине и $\exp (-\lambda \sigma(S))=\sigma(T(\lambda)) /\{0\} \forall \lambda \in \operatorname{Int} \Theta(\pi / 2-\alpha)$. Отсюда следует, что $-1 \in \rho(T(\lambda))$. Из $T(\lambda) \in \widetilde{C}$ и $(14)$ получаем

$$
\mathscr{R}\left(D_{T(\lambda)}\right)=\mathscr{R}\left(D_{T^{*}(\lambda)}\right)=\mathscr{R}\left(\left(I-T_{R}(\lambda)\right)^{1 / 2}\right)=\mathfrak{N} \quad \forall \lambda \in \operatorname{Int} \Theta(\pi / 2-\alpha)
$$

2) Положим в (15) $\lambda_{1}=\lambda_{2}=\lambda_{0}, T_{0}=T\left(\lambda_{0}\right), D_{T_{0}}=D_{T\left(\lambda_{0}\right)}, D_{T_{0}^{*}}=D_{T^{*}\left(\lambda_{0}\right)}$.

Из (15) $T(\lambda)=T_{0}+D_{T_{0}^{*}} \Psi(\lambda) D_{T_{0}}$, где $\Psi(\lambda)$ - голоморфная оператор-функция из $\mathscr{L}(\overline{\mathfrak{N}})$ в $\operatorname{Int} \Theta(\pi / 2-\alpha)$.

При любом $f \in H$ имеем $\forall \lambda_{1}, \lambda_{2} \in \operatorname{Int} \Theta(\pi / 2-\alpha)$

$$
g=\int_{\lambda_{1}}^{\lambda_{2}}(I-T(\lambda)) f d \lambda=\left(\lambda_{2}-\lambda_{1}\right)\left(I-T_{0}\right) f+D_{T_{0}^{*}}\left(\int_{\lambda_{1}}^{\lambda_{2}} \Psi(\lambda) D_{T_{0}} f d \lambda\right) .
$$

Как уже отмечалось, $\mathscr{R}\left(I-T_{0}\right) \subseteq \mathscr{R}\left(\left(I-T_{0 R}\right)^{1 / 2}\right)=\mathfrak{N}$. Значит $g \in \mathfrak{N}$.

ПРЕДЛОЖЕНИЕ 3. Для полугруппы $T(t)=\exp (-t S)$ nрu любыx $f \in H, u \in \mathscr{D}(s)$ $u 0<\tau<t$ справедливо неравенство

$$
|((T(\tau)-T(t)) f, u)|^{2} \leqslant \frac{1}{2 \cos ^{2} \alpha}(t-\tau)\left\|S_{R}^{1 / 2} u\right\|^{2}\left(\|T(\tau) f\|^{2}-\|T(t) f\|^{2}\right) .
$$


ДоказАтеЛЬСтво. Как известно [1], [2], $T(t) f \in \mathscr{D}(S) \forall f \in H, \forall t \in \operatorname{Int} \Theta(\pi / 2-\alpha)$. Справедливы также равенства [1], [2]:

$$
\begin{gathered}
T(\tau) f-T(t) f=\int_{\tau}^{t} S T(\xi) f d \xi \\
\|T(\tau) f\|^{2}-\|T(t) f\|^{2}=2 \int_{\tau}^{t}\left\|S_{R}^{1 / 2} T(\xi) f\right\|^{2} d \xi \quad \forall f \in H .
\end{gathered}
$$

Пусть $u \in \mathscr{D}(s)$, тогда из (5)

$$
\begin{aligned}
|((T(\tau)-T(t)) f, u)| & =\left|\left(\int_{\tau}^{t} S T(\xi) f d \xi, u\right)\right| \leqslant \int_{\tau}^{t}\left|\left(S_{R}^{1 / 2} T(\xi) f,(I-i G) S_{R}^{1 / 2} u\right)\right| d \xi \\
& \leqslant \frac{1}{\cos \alpha}\left\|S_{R}^{1 / 2} u\right\| \int_{\tau}^{t}\left\|S_{R}^{1 / 2} T(\xi) f\right\| d \xi \\
& \leqslant \frac{1}{\sqrt{2} \cos \alpha} \sqrt{t-\tau}\left\|S_{R}^{1 / 2} u\right\| \sqrt{\|T(\tau) f\|^{2}-\|T(t) f\|^{2}}
\end{aligned}
$$

Устремляя в (16) $\tau$ к +0 , получаем $\forall f \in H, \forall u \in \mathscr{D}(s)$

$$
|((I-T(t)) f, u)|^{2} \leqslant \frac{1}{2 \cos ^{2} \alpha} t\left\|S_{R}^{1 / 2} u\right\|^{2}\left\|D_{T(t)} f\right\|^{2}
$$

Полагая в (17) $f=u$, используя (12) и $\operatorname{Re}((I-T(t)) u, u) \leqslant|((I-T(t)) u, u)|$, получаем для любого $u \in \mathscr{D}(s)$

$$
\left\|\left(I-T_{R}(t)\right)^{1 / 2} u\right\|^{2} \leqslant|((I-T(t)) u, u)| \leqslant \frac{1}{\cos ^{2} \alpha}\left\|S_{R}^{1 / 2} u\right\|^{2} t
$$

Обозначим $A(t)=t^{-1}(I-T(t))$, тогда (18) можно переписать в виде

$$
\left\|A_{R}^{1 / 2}(t) u\right\|^{2} \leqslant|(A(t) u, u)| \leqslant \frac{1}{\cos ^{2} \alpha}\left\|S_{R}^{1 / 2} u\right\|^{2} \quad \forall u \in \mathscr{D}(s) .
$$

Из (19) $A_{R}^{1 / 2}(t) u=M(t) S_{R}^{1 / 2} u$, где $M(t) \in \mathscr{L}(H)$. Отсюда при любом $f \in H$ имеем $M^{*}(t) f \in \mathscr{D}(s)$ и $A_{R}^{1 / 2}(t) f=S_{R}^{1 / 2} M^{*}(t) f$.

Следовательно, при любом $t>0$

$$
\mathscr{R}\left(\left(I-T_{R}(t)\right)^{1 / 2}\right)=\mathfrak{N} \subseteq \mathscr{R}\left(S_{R}^{1 / 2}\right) .
$$


3. Доказательство теоремы 1. 2) $\Longrightarrow$ 1) - это теорема 3 с учетом

$$
\lim _{t \rightarrow+0} T(t) f=f \quad \forall f \in H .
$$

$3) \Longleftrightarrow 4)$ из $I-T(t) \in C(\alpha), t>0$, т.е. при любом $f \in H$

$$
\left\|\left(I-T_{R}(t)\right)^{1 / 2} f\right\|^{2} \leqslant|((I-T(t)) f, f)| \leqslant \frac{1}{\cos \alpha}\left\|\left(I-T_{R}(t)\right)^{1 / 2} f\right\|^{2} .
$$

1) $\Longrightarrow 3$ и и 1) $\Longrightarrow 4$ ) следует из (21) и (18).

$4) \Longrightarrow 2$ ). Пусть $t^{-1}\left\|\left(I-T_{R}(t)\right)^{1 / 2} u\right\|^{2} \leqslant C$ при $t>0$, где $C$ зависит от $u$.

Из (12) следует

$$
t^{-1}\left\|D_{T(t)} u\right\|^{2} \leqslant C .
$$

Рассмотрим функцию $u(t)=\left\|D_{T(t)} u\right\|^{2}=\|u\|^{2}-\|T(t) u\|^{2}$. Она непрерьвна на $[0, \infty)$ и дифференцируема на $(0, \infty)$, причем

$$
u^{\prime}(t)=2\left\|S_{R}^{1 / 2} T(t) u\right\|^{2}, \quad t>0, \quad u(0)=0 .
$$

По теореме Лагранжа $u(t)=u^{\prime}(\xi(t)) t$, где $\xi(t) \in(0, t)$.

Значит, $u(t)=2\left\|S_{R}^{1 / 2} T(\xi(t)) u\right\|^{2} t$. Из $(22)$ получаем $\left\|S_{R}^{1 / 2} T(\xi(t)) u\right\|^{2} \leqslant C$ при $t>0$. Следовательно, функция $|s[T(\xi(t)) u]|$ ограничена при $t \rightarrow+0$, т.е.

$$
\varliminf_{t \rightarrow+0}|s[T(t) u]|<\infty .
$$

1) $\Longrightarrow 6)$. Пусть $x \in \mathscr{D}(S)$, тогда из

$$
S x=\lim _{t \rightarrow+0} t^{-1}(I-T(t)) x, \quad\|x\|^{2}-\|T(t) x\|^{2}=\operatorname{Re}((I-T(t)) x,(I+T(t)) x)
$$

следует

$$
\lim _{t \rightarrow+0} t^{-1}\left\|D_{T(t)} x\right\|^{2}=2 \operatorname{Re}(S x, x)=2\left\|S_{R}^{1 / 2} x\right\|^{2} .
$$

Пусть теперь $u \in \mathscr{D}(s)$. Из (18) и $(12)$

$$
t^{-1}\left\|D_{T(t)} u\right\|^{2} \leqslant \frac{2}{\cos ^{2} \alpha}\left\|S_{R}^{1 / 2} u\right\|^{2} .
$$

Покажем, что

$$
\lim _{t \rightarrow+0} t^{-1}\left\|D_{T(t)} u\right\|^{2}=2\left\|S_{R}^{1 / 2} u\right\|^{2} .
$$

Так как $\mathscr{D}(S)$ ядро формы $s$, то для $u \in \mathscr{D}(s)$ и любого $\varepsilon>0$ найдется такой вектор $x \in \mathscr{D}(S)$, что выполняется неравенство $\left\|S_{R}^{1 / 2} u-S_{R}^{1 / 2} x\right\|<\varepsilon$. Из (23)

$$
\sqrt{t^{-1}}\left\|D_{T(t)} u-D_{T(t)} x\right\|<\sqrt{2} \frac{\varepsilon}{\cos \alpha} \text {. }
$$

Для $x \in \mathscr{D}(S)$ найдем $\delta=\delta(\varepsilon, x)$ такое, что при $t<\delta$ выполнено

$$
\left|\sqrt{t^{-1}}\left\|D_{T(t)} x\right\|-\sqrt{2}\left\|S_{R}^{1 / 2} x\right\|\right|<\varepsilon .
$$


Следовательно, при $t<\delta$ вьполняется

$$
\begin{aligned}
\left|\sqrt{2}\left\|S_{R}^{1 / 2} u\right\|-\sqrt{t^{-1}}\left\|D_{T(t)} u\right\|\right| \leqslant & \left|\sqrt{2}\left(\left\|S_{R}^{1 / 2} u\right\|-\left\|S_{R}^{1 / 2} x\right\|\right)\right| \\
& +\left|\sqrt{2}\left\|S_{R}^{1 / 2} x\right\|-\sqrt{t^{-1}}\left\|D_{T(t)} x\right\|\right| \\
& +\left|\sqrt{t^{-1}}\left(\left\|D_{T(t)} u\right\|-\left\|D_{T(t)} x\right\|\right)\right| \\
< & \left(1+\sqrt{2}+\frac{\sqrt{2}}{\cos \alpha}\right) \varepsilon .
\end{aligned}
$$

Поэтому

$$
\left.\frac{d}{d t}\left(\|T(t) u\|^{2}\right)\right|_{t= \pm 0}=-2\left\|S_{R}^{1 / 2} u\right\|^{2} .
$$

Отсюда следует существование $\left.(d / d t)(\|T(t) u\|)\right|_{t=0}$ и равенство (1).

$6) \Longrightarrow 2$ ). Из существования производной следует ограниченность функции $t^{-1}(\|u\|-\|T(t) u\|)$ при $t \rightarrow+0$. Следовательно, функция

$$
t^{-1}\left(\|u\|^{2}-\|T(t) u\|^{2}\right)=t^{-1}(\|u\|-\|T(t) u\|)(\|u\|+\|T(t) u\|)
$$

ограничена при $t \rightarrow+0$, т.е. справедливо (22). Далее проводим те же рассуждения, что и при доказательстве импликации 4$) \Longrightarrow 2$ ).

$5) \Longrightarrow 3)$. Из существования производной следует

$$
\sup _{t>0}\left|\left(t^{-1}(I-T(t)) u, u\right)\right|<\infty .
$$

1) $\Longrightarrow 5)$. Покажем, что для

$$
\left.\frac{d}{d t}(T(t) u, u)\right|_{t=+0}=-S[u] .
$$

Пусть $\omega[f, g]$ произвольная замкнутая плотно заданная полуторалинейная секториальная форма с вершиной в нуле и полууглом $\beta \in[0, \pi / 2), W_{R}$ - неотрицательньй самосопряженньй оператор, ассоциированный с $\omega_{R}$, тогда

$$
\omega[f, g]=\left((I+i \Gamma) W_{R}^{1 / 2} f, W_{R}^{1 / 2} g\right) \quad \forall f, g \in \mathscr{D}(\omega),
$$

где $\Gamma=\Gamma^{*} \in \mathscr{L}\left(\mathscr{R}\left(W_{R}\right)\right),\|\Gamma\| \leqslant \operatorname{tg} \beta$.

При $f, g \in \mathscr{D}(\omega)$ имеем

$$
\begin{aligned}
|\omega[f]-\omega[g]| & =|\omega[f-g, f]-\omega[g, g-f]| \\
& \leqslant\left|\left((I+i \Gamma) W_{R}^{1 / 2}(f-g), W_{R}^{1 / 2} f\right)\right|+\left|\left((I+i \Gamma) W_{R}^{1 / 2} g, W_{R}^{1 / 2}(f-g)\right)\right| .
\end{aligned}
$$

Следовательно, при всех $f, g \in \mathscr{D}(\omega)$

$$
|\omega[f]-\omega[g]| \leqslant \frac{1}{\cos \beta}\left\|W_{R}^{1 / 2}(f-g)\right\|\left(\left\|W_{R}^{1 / 2} f\right\|+\left\|W_{R}^{1 / 2} g\right\|\right) .
$$


Пусть $u \in \mathscr{D}(s)$. Тогда при любом $x \in \mathscr{D}(S)$

$$
\begin{aligned}
|(A(t) u, u)-s[u]| \leqslant & |(A(t) u, u)-(A(t) x, x)|+|(A(t) x, x)-(S x, x)| \\
& +|(S x, x)-s[u]|
\end{aligned}
$$

Применяя $(26)$ для форм $(A(t) u, u), s[u]$ и используя $(19)$, получаем

$$
\begin{gathered}
|(A(t) u, u)-s[u]| \leqslant \frac{1}{\cos \alpha}\left\|A_{R}^{1 / 2}(t)(u-x)\right\|\left(\left\|A_{R}^{1 / 2}(t) u\right\|+\left\|A_{R}^{1 / 2}(t) x\right\|\right) \\
\quad+|(A(t) x, x)-(S x, x)|+\frac{1}{\cos \alpha}\left\|S_{R}^{1 / 2}(u-x)\right\|\left(\left\|S_{R}^{1 / 2} u\right\|+\left\|S_{R}^{1 / 2} x\right\|\right) \\
\leqslant C\left\|S_{R}^{1 / 2}(u-x)\right\|\left(\left\|S_{R}^{1 / 2} u\right\|+\left\|S_{R}^{1 / 2} x\right\|\right)+|(A(t) x, x)-(S x, x)|
\end{gathered}
$$

причем $C$ не зависит от $t, u, x$.

Так как для $x \in \mathscr{D}(S)$ выполняется

$$
\lim _{t \rightarrow+0}(A(t) x, x)=(S x, x) \quad \text { и } \quad \inf \left\{\left\|S_{R}^{1 / 2}(u-x)\right\|, x \in \mathscr{D}(S)\right\}=0
$$

то получаем

$$
\lim _{t \rightarrow+0}(A(t) u, u)=s[u] .
$$

Следовательно, справедливо (25). Теперь равенства (2) и (3) получаются из (25) и (21) с использованием поляризованного тождества

$$
\omega[u, v]=\frac{1}{4}\{\omega[u+v]-\omega[u-v]+i \omega[u+i v]-i \omega[u-i v]\}
$$

верного для любой полуторалинейной формы $\omega$.

Из равенства

$$
t^{-1}\|(I-T(t)) u\|^{2}+t^{-1}\left\|D_{T(t)} u\right\|^{2}=2 \operatorname{Re}\left(t^{-1}(I-T(t)) u, u\right)
$$

при $u \in \mathscr{D}(s)$ из $(24)$ и $(25)$ получаем (4).

\section{4. Доказательство теоремы 2. Равенства}

$$
\mathscr{R}\left(D_{T(t)}\right)=\mathscr{R}\left(D_{T^{*}(t)}\right)=\mathscr{R}\left(\left(I-T_{R}(t)\right)^{1 / 2}\right)=\mathfrak{N}
$$

при всех $t \in \operatorname{Int} \Theta(\pi / 2-\alpha)$ уже установлены.

Пусть вектор $x \in \mathscr{R}\left(S_{R}^{1 / 2}\right)$. По предложению 1 имеем $|(x, u)| \leqslant C(x)\left\|S_{R}^{1 / 2} u\right\|$ для всех $u \in \mathscr{D}(s)=\mathscr{D}\left(S_{R}^{1 / 2}\right)$. Так как $T^{*}(\xi) f \in \mathscr{D}\left(S^{*}\right)$ для любого $f \in H, \xi \in \operatorname{Int} \Theta(\pi / 2-\alpha)$, то

$$
\left|\left(x, T^{*}(\xi) f\right)\right| \leqslant C(x)\left\|S_{R}^{1 / 2} T^{*}(\xi) f\right\| .
$$


Из (27) при $0<\tau<t$ получаем

$$
\begin{aligned}
\int_{\tau}^{t}|(T(\xi) x, f)| d \xi & \leqslant C(x) \int_{\tau}^{t}\left\|S_{R}^{1 / 2} T^{*}(\xi) f\right\| d \xi \\
& \leqslant C(x) \sqrt{t-\tau} \sqrt{\int_{\tau}^{t}\left\|S_{R}^{1 / 2} T^{*}(\xi) f\right\|^{2} d \xi} \\
& \leqslant C(x) \sqrt{t-\tau}\left(\frac{1}{2}\left(\left\|T^{*}(\tau) f\right\|^{2}-\left\|T^{*}(t) f\right\|^{2}\right)\right)^{1 / 2} \\
& \leqslant \frac{1}{\sqrt{2}} C(x) \sqrt{t-\tau}\left\|D_{T^{*}(t)} f\right\| .
\end{aligned}
$$

Так как

TO

$$
\left|\left(\int_{\tau}^{t} T(\xi) x d \xi, f\right)\right| \leqslant \int_{\tau}^{t}|(T(\xi) x, f)| d \xi
$$

$$
\left|\left(\int_{\tau}^{t} T(\xi) x d \xi, f\right)\right| \leqslant \frac{1}{\sqrt{2}} C(x) \sqrt{t-\tau}\left\|D_{T^{*}(t)} f\right\| .
$$

Из предложения 1 и (28) следует

$$
h=\int_{\tau}^{t} T(\xi) x d \xi \in \mathscr{R}\left(D_{T^{*}(t)}\right)=\mathfrak{N} .
$$

По предложению 2 вектор

$$
g=\int_{\tau}^{t}(I-T(\xi)) x d \xi
$$

лежит в $\mathfrak{N}$. Поскольку $h=-g+(t-\tau) x$, то $x \in \mathfrak{N}$.

Таким образом, $\mathscr{R}\left(S_{R}^{1 / 2}\right) \leqslant \mathfrak{N}$. Противоположное включение (20) уже установлено.

Отметим, что

1) $T(t) x \in \mathscr{R}\left(S_{R}^{1 / 2}\right), t \in \operatorname{Int} \Theta(\pi / 2-\alpha)$ тогда и только тогда, когда $x \in \mathscr{R}\left(S_{R}^{1 / 2}\right)$;

2) $\int_{\tau}^{t} T(\xi) x d \xi \in \mathscr{R}\left(S_{R}^{1 / 2}\right)$ тогда и только тогда, когда $x \in \mathscr{R}\left(S_{R}^{1 / 2}\right)$.

Эти утверждения следуют из равенств $T(t) x=x-(I-T(t)) x$,

$$
\int_{\tau}^{t} T(\xi) x d \xi=(t-\tau) x-\int_{\tau}^{t}(I-T(\xi)) x d \xi
$$

предложения 2 и теоремы 2.

Кроме того, из (16) и предложения 1 при любом $f \in H$

$$
\begin{gathered}
(T(t)-T(\tau)) f \in \mathscr{R}\left(S_{R}^{1 / 2}\right), \quad t, \tau \in \operatorname{Int} \Theta(\pi / 2-\alpha), \\
\left\|S_{R}^{-1 / 2}(T(t)-T(\tau) f)\right\|^{2} \leqslant \frac{1}{2 \cos ^{2} \alpha}(t-\tau)\left(\|T(\tau) f\|^{2}-\|T(t) f\|^{2}\right), \quad 0<\tau<t .
\end{gathered}
$$

Отсюда следует, что при $x \in \mathscr{R}\left(S_{R}^{1 / 2}\right)$ вектор-функция $S_{R}^{-1 / 2} T(t) x$ сильно непрерывна на $[0, \infty)$ и

$$
\left\|S_{R}^{-1 / 2} x-S_{R}^{-1 / 2} T(t) x\right\|=o(\sqrt{t}), \quad t \rightarrow+0 .
$$




\section{СПИСОК ЦИТИРОВАННОЙ ЛИТЕРАТУРЫ}

[1] Като Т. Теория возмущений линейных операторов. М.: Мир, 1972.

[2] Крейн С. Г. Линейные дифференциальные уравнения в банаховом пространстве. М.: Наука, 1967.

[3] Клемент Ф., Хейманс Х., Ангенент С., ван Дуйн К., де Пахтер Б. Однопараметрические полугруппы. М.: Мир, 1992.

[4] Крейн С. Г. Теория самосопряженных расширений полуограниченных эрмитовых операторов и ее приложения // Матем. сб. 1947. Т. 20. № 3. С. 431-490.

[5] Шмульян Ю.Л. Двустороннее деление в кольце операторов // Матем. заметки. 1967. T. 1. №5. C. $605-610$.

[6] Ando T., Nishio K. Positive self-adjoint extensions of positive symmetric operators // Tôhoku Math. J. 1970. V. 22. P. 65-75.

[7] Арлинский Ю. М. Об одном классе сжатий в гильбертовом пространстве // Укр. матем. ж. 1977. Т. 39. №6. С. 691-696.

[8] Сёкефальви-Надь Б., Фолш Ч. Гармонический анализ операторов в гильбертовом пространстве. М.: Мир, 1970.

[9] Fillmore P. A., Williams J. P. On operator ranges // Adv. Math. 1971. V. 7. P. 254-281.

[10] Шмульян Ю. Л. О некоторых свойствах стабильности для аналитических оператор-функций // Матем. заметки. 1976. Т. 20. № 4. С. 511-520. 